\title{
Médiévales
}

Langues, Textes, Histoire

\section{L'art et le théâtre au Moyen Âge : jalons et perspectives}

Art and Theatre in the Middle Ages - Pointers and Perspectives

\section{Rose-Marie Ferré}

\section{(2) OpenEdition}

\section{Journals}

Édition électronique

URL : https://journals.openedition.org/medievales/6079

DOI : 10.4000/medievales. 6079

ISSN : $1777-5892$

Éditeur

Presses universitaires de Vincennes

Édition imprimée

Date de publication : 31 décembre 2010

Pagination : 77-89

ISBN : 978-2-84292-267-2

ISSN : 0751-2708

\section{Référence électronique}

Rose-Marie Ferré, «L'art et le théâtre au Moyen Âge : jalons et perspectives », Médiévales [En ligne], 59 | automne 2010, mis en ligne le 20 mars 2013, consulté le 23 avril 2022. URL : http://

journals.openedition.org/medievales/6079; DOI : https://doi.org/10.4000/medievales.6079 
Médiévales 59, automne 2010, p. 77-89

Rose-Marie FERRÉ

\section{L'ART ET LE THÉÂTRE AU MOYEN ÂGE: JALONS ET PERSPECTIVES}

Il revient à Émile Mâle d'avoir pour la première fois abordé la question de la relation entre les arts figurés et le théâtre. Ce spécialiste a défendu en effet une thèse de large portée supposant l'influence de l'art dramatique et de ses procédés sur les arts. Même si cette réflexion est aujourd'hui jugée comme trop radicale, il faut cependant rendre justice aux intuitions de ce grand penseur.

Force est aussi de constater que ce domaine de recherche est resté longtemps problématique. Les approches ont été diverses: certains chercheurs se sont réclamés d'É. Mâle tandis que d'autres s'y sont opposés farouchement. Mais, malgré les polémiques, l'indifférence aussi, les interprétations erronées que l'on a faites de ses écrits, É. Mâle a ouvert de nombreuses pistes de recherche. Fonctionnalité des images, dialogue entre les arts, contexte de réception des œuvres, «médialité médiévale» : d'autres voies d'investigation ont vu le jour. Il s'agit donc ici de faire le bilan de ces études et de montrer tout l'intérêt d'une interrogation conjointe de plusieurs modes d'expression artistique.

Si les relations de l'art dramatique et des arts figurés ont intéressé É. Mâle, il convient de revenir en premier lieu, comme l'ont inauguré les auteurs du volume collectif consacré au penseur en $2005^{1}$, sur la démarche plus générale de ce grand intellectuel, premier chargé de cours d'histoire de l'art chrétien du Moyen Ầge à l'Université de la Sorbonne en 1906, puis titulaire de la chaire d'histoire de l'art en 1912.

Conséquence de la loi de séparation des Églises et de l'État de 1905, la suppression de la Faculté d'État de Théologie Protestante a laissé en effet la place à de nouveaux enseignements à l'Université. Le cours dont É. Mâle prend alors la charge s'inscrit dans cette démarche laïque qui fait entrer l'étude des arts chrétiens

1. A. VAuchEZ dir., Émile Mâle (1862-1954), la construction de l'œuvre: Rome et l'Italie, Actes de la table ronde qui s'est tenue à l'École Française de Rome les 17 et 18 juin 2002, Rome, 2005. 
dans le domaine des sciences historiques. Le sujet de thèse d’É. Mâle (déposé en 1892) est d'ailleurs nouveau - l'étude de l'art chrétien médiéval (xIII siècle) -, et ses méthodes d'investigation le sont tout autant: examen critique des documents du passé (sources d'archives et textes théologiques), observation minutieuse des monuments, avec relevés, et analyse sérielle des images.

La composition de son jury de thèse est aussi révélatrice de ce nouveau mode d'enquête qui croise différentes spécialités: ainsi le président, Georges Perrot (1839-1914), directeur de l'École Normale Supérieure et spécialiste d'Antiquité grecque et d'archéologie, ou Charles Victor Langlois (1863-1929), professeur de paléographie et d'histoire du Moyen Âge, futur directeur de l'École des chartes, ou enfin Louis Petit de Julleville (1841-1900), titulaire de la chaire de littérature française du Moyen Âge et d'histoire de la langue française à l'université de la Sorbonne et auteur d'un des premiers ouvrages consacrés au théâtre français du Moyen Âge. Dans cette perspective, les thématiques des enseignements retenues par É. Mâle relèvent pareillement du vaste champ de l'histoire religieuse et suggèrent son intérêt pour l'évolution des idées, comme en témoignent par ailleurs ses quatre grands ouvrages de synthèse $(1898,1908,1922,1932)$.

Toutefois, et en dépit des efforts d'É. Mâle pour faire ressortir l'individualité de la création et de l'artiste, l'art médiéval n'est envisagé que comme un système rigide de signes univoques. De même, les sources écrites sont parfois utilisées mécaniquement pour expliquer le sens des images. Peu d'exemples précis sont finalement présentés, et cela à dessein par souci de synthèse. É. Mâle avouera lui-même avoir voulu constamment «aller à la rencontre de l'esprit» et de la pensée organisatrice propres à tout homme et à ses œuvres. Audacieuse entreprise, certains aspects de cette réflexion doivent à présent être examinés comme la question de l'«influence du théâtre sur l'art à la fin du Moyen Âge».

Ce sujet a été abordé pour la première fois par É. Mâle lors d'une lecture faite à l'Académie des Inscriptions et Belles-Lettres, durant la séance du 31 juillet $1903^{2}$. Se fondant toujours sur la recherche des sources littéraires, il envisage d'analyser certains motifs iconographiques majeurs des XIV et $\mathrm{XV}^{\mathrm{e}}$ siècles à la lumière des témoignages dramatiques. Les quatre articles capitaux intitulés «Le renouvellement de l'art par les mystères à la fin du Moyen Âge» publiés dans la Gazette des Beaux-Arts en 1904 couronnent cette démarche ${ }^{3}$.

2. Analysée dans les Comptes rendus des séances, 1903, p. 330.

3. É. MÂLE, «Le renouvellement de l'art par les mystères à la fin du Moyen Âge», Gazette des Beaux-Arts, $3^{\text {e }}$ s., 31, février 1904, p. 89-106; mars, p. 215-230; avril, p. 283-301 ; mai, p. 379394 [repris dans ID., L'Art religieux de la fin du Moyen Âge en France. Étude sur l'iconographie du Moyen Âge et sur ses sources d'inspiration, Paris, 1908, chap. I, p. 34-74]. D'autres essais de l'auteur reprennent cependant cette thématique: «Influence du théâtre italien sur l'art italien du Xv $v^{\mathrm{e}}$ siècle», Gazette des Beaux-Arts, $3^{\mathrm{e}}$ série, 35, février 1906, p. 89-94. Voir aussi ses réflexions sur les drames liturgiques et les arts: ID., «Les influences du drame liturgique sur la sculpture 
Dans un premier temps l'auteur se demande «comment l'iconographie chrétienne, qui s'était développée jusque-là avec autant de lenteur que le dogme, a-t-elle pu se transformer si brusquement?» Il répond en affirmant que «ce changement s'explique par l'épanouissement du théâtre religieux dans la Chrétienté tout entière au commencement du $\mathrm{Xv}^{\mathrm{e}}$ siècle $^{4}{ }$. Mais É. Mâle montre surtout que les auteurs dramatiques se sont inspirés essentiellement des Méditations sur la vie de Jésus-Christ du pseudo-saint Bonaventure pour créer de nouvelles scènes, plus pittoresques et dynamiques. Ce n'est qu'ensuite, par l'intermédiaire des mystères, que l'ancienne iconographie s'est renouvelée. Déjà, l'auteur remarque que la pensée franciscaine a pu enrichir le jeu théâtral ${ }^{5}$.

Dans son deuxième article, É. Mâle insiste sur le fait que les artistes, qui assistaient aux représentations théâtrales et qui y collaboraient le plus souvent, s'inspiraient directement pour leurs œuvres des scènes qu'ils avaient vu jouer. S'appuyant sur plusieurs exemples - Procès de Paradis, Annonciation, Passion -, il souligne dès lors la primauté du drame sur les arts figurés: "On peut dire de toutes les scènes nouvelles, qui entrent alors dans l'art plastique, qu'elles ont été jouées avant d'être peintes ${ }^{6}$.» Fidèle à ses premières amours, É. Mâle en arrive même à considérer que, par le biais des Méditations sur la vie de Jésus-Christ, «c'est le génie du XIII ${ }^{e}$ siècle qui a vivifié la littérature et l'art du $\mathrm{XV}^{\mathrm{e}}$ siècle» ${ }^{7}$ !

Le troisième article explore aussi les épisodes et les agencements iconographiques inédits que le texte du pseudo-saint Bonaventure ne peut expliquer. É. Mâle passe alors en revue les événements de la vie de Jésus-Christ, en mettant en évidence tout ce que l'art doit à l'influence directe des mystères (par exemple les personnages nouveaux des sages-femmes pendant la Nativité, l'Adoration des Bergers ou encore la scène de la Véronique lors du Portement de Croix - thème que l'on retrouve en fait dans la littérature aprocryphe). Il affirme que «les mystères ont mis sous les yeux des artistes des scènes de la vie de Jésus-Christ auxquelles ils n'avaient jamais pensé, et leur ont donné l'idée de les représenter ${ }^{8}$ ». Les artistes ont aussi emprunté aux mystères les costumes des personnages. Allant plus loin, l'historien de l'art voit dans le nouveau réalisme du $\mathrm{Xv}^{\mathrm{e}}$ siècle la marque du drame. Prenant l'exemple de la Crucifixion, dorénavant dépouillée de tout symbolisme, il conclut que «ce désir de représenter un fait dans sa vérité historique n’a

romane», Revue de l'art ancien et moderne, 22, août 1907, p. 81-92, ou ID., «Le drame liturgique et l'iconographie de la Résurrection», Revue de l'art ancien et moderne, 39, avril 1921, p. 213 222: É. Mâle établit en effet un puissant rapprochement entre l'art et la liturgie puisqu'il considère la liturgie comme le domaine par excellence de la pensée symbolique de la période médiévale.

4. É. MÂLE, op. cit., 1904, p. 95-96.

5. Ibid., p. 106.

6. Ibid., p. 215 .

7. Ibid., p. 230 .

8. Ibid., p. 289. 
pas dû venir d'abord aux artistes» et qu'il faut l'imputer «aux organisateurs des représentations théâtrales ${ }^{9}$.

Enfin, dans le dernier volet de sa réflexion, après avoir abordé les éléments de décors et accessoires que les artistes ont imités de la mise en scène théâtrale, É. Mâle se livre à une conclusion édifiante sur les apports du théâtre à l'art français de la fin du Moyen Âge. Non dénué d'intérêt historiographique à cause des problèmes qu'il soulève, nous reproduisons ici le passage terminant les démonstrations d'É. Mâle:

On a beaucoup écrit sur les Mystères. On a longuement disserté sur l'art des poètes dramatiques, sur leur style, sur leur versification, - toutes choses de peu de prix. On a tout dit des Mystères, sauf pourtant l'essentiel. Car, s'ils méritent l'attention, ce n'est point du tout à cause de leurs qualités littéraires : ce sont de pauvres choses que nos drames religieux du $\mathrm{XV}^{\mathrm{e}}$ siècle. Mais comment leur être sévère quand on songe qu'ils ont inspiré les maîtres les plus exquis ? N'est-ce rien d'avoir renouvelé des sujets que trois siècles avaient consacrés, d'avoir (merveille inouïe) enfanté un nouvel art chrétien qui vivra deux cents ans? N'est-ce rien d'avoir enseigné à van Eyck, à Fouquet, à Memling, à Dürer, des groupements, des attitudes, de leur avoir imposé des costumes et jusqu'à la couleur de ces costumes? Cela revient à dire que, dans le théâtre du moyen âge, le tableau vivant a seul de l'intérêt. [...] Les érudits se sont posé mille questions sur la mise en scène des Mystères, comme si c'était là un problème difficile à résoudre. Il suffit de regarder. Les tableaux, les vitraux, les miniatures, les retables nous offrent sans cesse l'image exacte de ce qu'on voyait au théâtre. Certaines œuvres d'art sont des copies plus frappantes encore, car l'action y est simultanée, comme dans les Mystères. Les tableaux de Memling consacrés à la Passion et à la vie de la Vierge - où l'on voit dix scènes différentes se dérouler sur le même fond de paysage, où les acteurs du drame se transportent naïvement d'une mansion à une autre - nous donnent l'idée la plus exacte d'une représentation dramatique ${ }^{10}$.

Proche des préoccupations qui animaient alors les historiens de l'art français, et dont témoigne l'exposition des Primitifs français organisée par Henri Bouchot la même année, É. Mâle met en exergue un fort sentiment national faisant de la France, grâce au théâtre des mystères, le foyer de renouvellement de l'iconographie religieuse ${ }^{11}$ ! É. Mâle sera toutefois plus nuancé sur la question du rapport des arts figurés et du théâtre dans ses écrits ultérieurs ${ }^{12}$.

Loin de n'être qu'une formule obligée, force est pourtant de constater que, si les travaux d'É. Mâle sur l'influence du drame sur les arts figurés sont incontournables, il n'en demeure pas moins qu'ils suscitent de graves débats et présen-

9. Ibid., p. 296.

10. Ibid, p. 390.

11. Ibid., p. 392-394.

12. É. MÂLE, op. cit., 1908 (2édition revue et augmentée, 1922), p. 35-85. 
tent des limites certaines. Les difficultés générées par ses réflexions concernent autant l'histoire du théâtre que celle de l'art médiéval, sur lequel nous insisterons. Plus largement, la principale aporie présentée par cette thèse est celle d'un raisonnement en termes d'ascendant, et donc d'antériorité et de hiérarchie d'un mode d'expression artistique sur un autre: «Toutes les scènes nouvelles, qui entrent alors dans l'art plastique [du $\mathrm{Xv}^{\mathrm{e}}$ siècle] [...] ont été jouées avant d'être peintes ${ }^{13}$.» Que ce soit les attitudes de l'ange et de la Vierge de l'Annonciation qui d'une position hiératique vont adopter sous l'impact des innovations dramatiques les postures de la salutation et de la génuflexion, ou que ce soit les nouvelles scènes pathétiques du cycle de la Passion (les adieux de Marie à son fils ou la scène de la Vierge de Pitié insérée entre la descente de croix et la mise au tombeau), É. Mâle démontre toujours la prévalence du drame ${ }^{14}$. Il franchit cependant la limite de la véracité scientifique en prenant l'exemple de l'apparition du motif de la colonne sur laquelle s'appuie la Vierge avant d'enfanter ${ }^{15}$. S'il accepte qu'aucune trace de cet épisode, inventé et développé encore une fois par le pseudo-saint Bonaventure, n'existe dans le théâtre, É. Mâle voit dans son avènement dans la peinture (flamande notamment) l'influence directe des mystères! Or, l'auteur nie ici le fait que les artistes puissent avoir eu recours directement au texte des Méditations pour concevoir de nouvelles scènes et ne veut pas accepter la moindre faille dans le système de dépendance qu'il a élaboré.

Comme le souligne Alois Maria Nagler en 1976 dans un chapitre de son ouvrage sur la mise en scène consacré aux relations des arts figurés et du théâtre ${ }^{16}$, le problème principal qui a ensuite préoccupé la critique a été de savoir qui, du théâtre ou des arts visuels, a eu la primauté des innovations iconographiques de la fin du Moyen Âge. Plusieurs courants de pensée se sont alors dessinés dans l'historiographie.

Premièrement, un certain nombre de chercheurs, dont Gustave Cohen, Hildeburgh, Grace Frank ou Otto Pächt par exemple, ont poursuivi fidèlement les positions d'É. Mâle sur l'antériorité du théâtre sur l'iconographie ${ }^{17}$.

13. É. MÂLE, op. cit., 1904, p. 215.

14. Ibid., p. 220-227.

15. Ibid., p. 221.

16. A.M. NAGLer, The Medieval Religious Stage. Shapes and Phantoms, New Haven Londres, 1976, chap. 6, p. 89-105.

17. G. CoHEn, Histoire de la mise en scène dans le théâtre religieux français du Moyen Âge, $3^{e}$ édition, Paris, 1951; G. Frank, The Medieval French Drama, Oxford, 1954; W.L. HiLdeburgh, «English Alabaster Carvings as Records of the Medieval English Drama», Archaoologia, 93, 1955, p. 51-101; O. Рёснт, The Rise of Pictorial Narrative in Twelfth-Century England, Oxford, 1962. Voir aussi: A. RAPP, Studien über den Zusammenhang des geistlichen Dramas mit der bildenden Kunst, Munich, 1936, ou M.D. ANDERson, Drama and imagery in english Medieval Churches, Cambridge, 1963. 
Une deuxième thèse consiste, au contraire, à penser qu'à cause des traditions byzantines, l'art graphique avait joui d'une antériorité temporelle et exercé une influence sur les mystères ${ }^{18}$. Enfin, d'autres courants plus nuancés estiment que l'art et le théâtre sont fondés sur une littérature commune (sources apocryphes, Légende dorée de Jacques de Voragine, textes mystiques, littérature pastorale) et connaissent donc une évolution parallèle qui empêche tout raisonnement en terme d'influence d'un art sur un autre ${ }^{19}$.

Si ces approches présentent par conséquent le défaut de l'univocité, il convient cependant - tant pis pour le truisme ! - de les envisager toutes à la fois eu égard à l'ambivalence et l'ambiguïté de l'art du Moyen Âge. Voici le principal handicap des conceptions d'É. Mâle et des controverses qu'elles ont engagées. Dans un souci revendiqué de systématisation, É. Mâle n'apprécie pas à sa juste valeur le caractère «inter-référent» des arts de la période médiévale, et plus particulièrement du $\mathrm{Xv}^{\mathrm{e}}$ siècle. D'ailleurs, le concept d'«influence», litigieux tant il est imprécis et inadapté aux investigations sur l'art du Moyen Âge, met de côté non seulement le contexte précis de création des œuvres et les questions relatives à leurs fonctions, mais évacue aussi toute réflexion sur les paramètres de la commande artistique et sur le travail et le langage esthétique de chaque artiste.

Le dernier exemple pris par É. Mâle est ainsi évocateur; il concerne la simultanéité des actions du jeu théâtral que l'on retrouve dans la peinture. Selon lui «les tableaux de Memling consacrés à la Passion et à la vie de la Vierge où l'on voit dix scènes différentes se dérouler sur le même fond de paysage, où les acteurs du drame se transportent naïvement d'une mansion à une autre nous donnent l'idée la plus exacte d'une représentation dramatique ${ }^{20}$. Peu de prix est ici accordé à la liberté créatrice de l'artiste et aux processus de création des images qui, quand ils sont compris, prouvent que la peinture a ses règles et son langage propre. Et de même, peu de cas est fait aux exercices de méditation entretenus par l'image. C'est ce qui a d'ailleurs été dommageable à l'interprétation de la fameuse miniature du Martyre de saint Apolline peinte par Jean Fouquet dans le Livre d'heures d'Étienne Chevalier dans laquelle on a voulu voir absolument la retranscription exacte d'une scène de mystère ${ }^{21}$. Les récentes études de Nicole Reynaud rectifient cette vision, même si l'on ne peut pas

18. Par exemple: F.P. Pickering, Literature and Art in the Middle Ages, Coral Gables, Fla. (traduction anglaise de la version allemande), 1970. À partir de la deuxième édition de son Art religieux de la fin du Moyen Âge... (1922), Mâle prendra également en considération les apports de l'art byzantin et nuancera ses propos sur les rapports du drame et des arts.

19. E. GRUBE, «Die abendländisch-christliche Kunst des Mittelalters und das geistliche Schauspiel der Kirche», Maske und Kothurn, 3, 1957, p. 22-59 (p. 57).

20. É. MÂLE, op. cit., 1904, p. 390.

21. Chantilly, Musée Condé, miniature faisant partie des suffrages des saints. 
nier une référence explicite au théâtre, mais elles prouvent aussi que l'artiste répondait avant tout aux impératifs de la peinture et non à ceux du théâtre ${ }^{22}$.

Le thème des arts dans le théâtre ou du théâtre dans les arts semble en tout cas constituer aujourd'hui un nouveau défi pour l'historiographie. Déjà dans leurs travaux fondateurs, Erwin Panofsky et Pierre Francastel avaient inauguré de nouvelles conceptions de la création artistique, tributaire de son environnement politique, social et culturel ${ }^{23}$. C'est la démarche que l'on retrouve dans le domaine de l'anthropologie historique et chez certains historiens des images comme Hans Belting, Jean Wirth, Jean-Claude Schmitt ou Jérôme Baschet ${ }^{24}$.

Mais, au-delà de ces approches théoriques, les liens entre théâtre et peinture ont été abordés par certains historiens de l'art. Bernard Mosse s'est interrogé sur la notion de théâtralité picturale à travers le concept de narrativité au XIV ${ }^{\mathrm{e}}$ siècle ${ }^{25}$. D'autre part, certains aspects concrets du théâtre ont intéressé ponctuellement les chercheurs. Les études sur les décors de fêtes soulignent par exemple l'étendue de l'intervention des artistes dans la mise en scène et apportent déjà des pistes de réflexion sur les processus de création des images ${ }^{26}$. Elles ne sont malheureusement ni reprises ni prolongées.

22. N. Reynaud, Jean Fouquet. Les Heures d'Etienne Chevalier, Paris, 2006, p. 216-222. Voir aussi la récente mise au point de V. DominguEZ, «La scène et l'enluminure: 1'Apolline de Jean Fouquet dans le Livre d'Heures d'Étienne Chevalier», Romania, t. 122, 2004 (3-4), p. 468-505.

23. E. PANofsKy, L'Euvre d'art et ses significations: essais sur les arts visuels, (traduction française de l'anglais), Paris, 1969, ou ID., Peinture et dévotion en Europe du Nord à la fin du Moyen Âge, présentation par Daniel Arasse, Paris, 1997. P. FRANCASTEL, La Réalité figurative: éléments

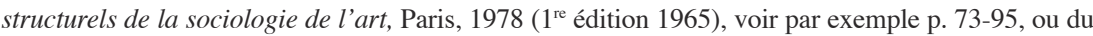
même auteur: La Figure et le lieu: l'ordre visuel du Quattrocento, Paris, 1980 (1 $1^{\mathrm{re}}$ édition 1967). Penser également aux réflexions de M. BAXANDALl: L'Eil du quattrocento. L'usage de la peinture dans l'Italie de la Renaissance (traduction de l'anglais), Paris, 1985.

24. H. Belting, L'image et son public au Moyen Âge (traduction de l'allemand), Paris, 1998, ou Image et culte : une histoire de l'image avant l'époque de l'art (traduction de l'allemand), Paris, 1998 ; J. Wirth, L'Image médiévale. Naissance et développements (VI'-XVe siècle), Paris, 1989, ou bien encore J. BASChet, J. CL. SchmitT dir., L'Image : fonctions et usages des images dans l'Occident médiéval, Paris, 1996.

25. B. Mosse, «De la théâtralité de la peinture au XIV ${ }^{\mathrm{e}} \mathrm{s}$. D'Assise à Avignon : théâtre et peinture», dans Théâtre et spectacles hier et aujourd'hui. Moyen Âge et Renaissance, Actes du $115^{\circ}$ Congrès national des sociétés savantes, Section d'histoire médiévale et de philologie, Avignon, 1990, Paris, 1991, p. 83-92.

26. Par exemple: A. MéLıcoQ, «Artistes des $\mathrm{XV}^{\mathrm{e}}$ et $\mathrm{XVI}$ e siècles qui ont orné les hourds des joueurs par personnages, etc.», La Revue de l'Art, 21, 1865, p. 255-260 ; D. EICHBERGER, «The tableau vivant - an Ephemeral Art Form in Burgundian Civic Festivities», Parergon. Australian and New Zealand association for medieval and Renaissance studies, 6A, 1988, p. 37-64; F. JOUBERT, «Les peintres du Vœu du Faisan», dans M. Th. CARon, D. Clauzel dir., Le Banquet du Faisan. 1454, l'Occident face au défi de l'Empire ottoman, Arras, 1997, p. 187-200, ou EAD., «Le Mariage de Charles le Téméraire et Marguerite d'York et ses enjeux artistiques», dans Art et transferts culturels au temps de Charles le Téméraire (sous presse). L'intervention des peintres lors de la création des spectacles est aussi connue 
Il faudrait aussi se tourner vers les récentes analyses des implications des œuvres d'art dans les pratiques dévotionnelles ou liturgiques comme l'a fait le récent ouvrage dirigé par Francesca Flores d'Arcais, consacré à la théâtralité de la sculpture italienne ${ }^{27}$, ou encore les importants travaux concernant les retables siennois $^{28}$.

Plus spécifiques aux études théâtrales, certains spécialistes de la littérature ont essayé dans le même temps de montrer toute la pertinence de l'analyse conjointe des arts figurés et de l'histoire du théâtre. Cette nouvelle approche a été promue en particulier par toute une série de volumes intitulés Early Drama, Art and Music du Medieval Institute de la Western Michigan University. Les travaux de Clifford Davidson, et notamment son Drama and Art de 1977, ont inauguré une nouvelle méthode de recherche pluridisciplinaire ${ }^{29}$. L'auteur a cherché à savoir ce que l'histoire de l'art pouvait apporter à la connaissance de l'histoire du théâtre. Il envisage les arts visuels comme des outils indispensables à la compréhension du drame médiéval. Certes réductrice, cette position permet cependant d'imaginer une relation du théâtre et des arts en termes d'échanges, mettant fin par là même à toute conception hiérarchique dans la veine d'É. Mâle.

Martin Stevens nuance néanmoins cette tentative. Il propose notamment de réexaminer la relation entre l'art dramatique et les arts figurés en dépassant, d'une part, l'interprétation causale (l'influence d'un art sur un autre) et, d'autre part, la proposition de Clifford Davidson de n'utiliser les arts que comme des outils pour le théâtre. Analysant le célèbre retable de la Passion de Hans Memling (Turin), Stevens veut démontrer l'intertextualité de deux moyens d'expression artistique de la fin du Moyen Âge ${ }^{30}$.

dans les monographies consacrées aux artistes: voir par exemple les travaux d'Élisabeth Dhanens sur Hugo van der Goes.

27. F. Flores D'ArCaIs dir., Il teatro delle statue. Gruppi lignei di Deposizione e Annunciazione tra XII e XIII secolo, Milan, 2005. Consulter aussi: M. BuRRESI dir., Sacre Passioni. Scultura lignea a Pisa dal XII al XV secolo, Milan, 2000.

28. Entre autres: K. VAN DeR Ploeg, «How liturgical is a medieval altarpiece?», dans V.M. SснміDт éd., Italian panel painting of the Duecento and Trecento, Studies in the history of art, 61, 2002, p. 102-121; P. Crossley, «The Man from Inner Space: Architecture and Meditation in the Choir of St Laurence in Nuremberg», Medieval Art - Recent Perspectives : A Memorial Tribute to C.R. Dodwell, Manchester, 1998, ou B. Williamson, «Altarpieces, Liturgy and Devotion», Speculum, t. 79/2, 2004, p. 341-406.

29. C. DAVIDSON, Drama and Art: An Introduction to the Use of Evidence from the Visual Arts for the Study of Early Drama, Early Drama, Art, and Music Monograph Series, 1, Kalamazoo, 1977, en particulier chap. 1: «Drama and Art», p. 1-14, et le chap. 9: «Interdisciplinary Criticism and Medieval Drama», p. 100-125.

30. M. Stevens, «The Intertextuality of Late Medieval Art and Drama», New Literary History, 22, 1991, p. 317-337. 
Cette méthode prolonge finalement celle de Pamela Sheingorn qui, dans ses nombreux essais, a montré toute l'importance de la prise en compte de la réciprocité des arts visuels et performatifs ${ }^{31}$. Ses travaux portent aussi sur les problématiques du manuscrit et du rapport texte-image. En particulier, son article rédigé en collaboration avec Robert Clark sur les manuscrits illustrés du mystère de la Passion d'Arnoul Gréban pose la question fondamentale des pratiques de lecture et de réception ${ }^{32}$. Ces réflexions apportent en effet de nouveaux éléments de compréhension sur les relations des différents langages artistiques - éphémères ou pérennes ${ }^{33}$. Cette démarche, ainsi adoptée, dans le cadre de recherches récentes, pour l'étude de diverses œuvres d'art, a le mérite de mettre justement en valeur le caractère parfois «intermédiatique» de la création artistique médiévale ${ }^{34}$.

L'exemple du retable du Portement de Croix que le sculpteur Francesco Laurana a exécuté pour le roi René en 1478, et destiné à orner l'autel majeur de l'église des Célestins d'Avignon, est à ce titre particulièrement révélateur ${ }^{35}$ (Fig. 1). En effet, son iconographie a longtemps interpellé les chercheurs qui,

31. Par exemple, se référer à P. SHEINGorn, «On Using Medieval Art in the Study of Medieval Drama: an Introduction to Methodology», Research Opportunities in Renaissance Drama, 22, 1979, p. 101-109; « The Visual Language of Drama: Principles of Composition», dans M. BRISCOE, J.C. Coldewey dir., Contexts for Early English drama, Bloomington, 1989, p. 173-191, ou «Medieval Studies and the New Art History », Mediaevalia, 18, 1995, p. 143-162.

32. R.L.A. Clark, P. Sheingorn, «Performative Reading: The Illustrated Manuscripts of Arnoul Gréban's Mystère de la Passion», European Medieval Drama, 6, 2002, p. 129-154, ou «Were Guillaume de Digulleville's Pèlerinage "Plays"? The Case for Arras 845 as Performative Anthology», ibid., 12, 2008, p. 109-147. Voir aussi P. SHEINGORN, «Illustrations in the Manuscript of the Lille Plays», Research Opportunities in Renaissance Drama, 30, 1988, p. 173-176, ou M. Cruse, G. Parussa, I. Ragnard, «The Aix Jeu de Robin et Marion: Image, Texte, Music », Studies in Iconography, 25, 2004, p. 1-46.

33. Se référer également à L. Weigert, «Velum Templi: Painted Cloths of the Passion and the Making of Lenten Rituals in Reims», Studies in Iconography, 24, 2003, p. 199-229, et «Illuminating the Arras Mystery Play», dans N.A. Rowe, D.S. Areford dir., Excavating the Medieval Image. Manuscripts, Artists, Audiences: Essays in Honor of Sandra Hindman, Aldershot, 2004, p. 81-108; puis aux travaux d'E. GERTSMAN, «Pleyinge and Peyntynge: Performing the Dance of Death», Studies in Iconography, 27, 2006, p. 1-43, et ID., Visualizing Medieval Performance. Perspectives, Histories, Contexts, Aldershot, 2008.

34. R.-M. FerRé, La Commande artistique à la cour de René d'Anjou : un concert de mots et d'images, Thèse de l'université de Paris IV-Sorbonne, 2008.

35. Pour une étude détaillée de cette œuvre, voir R.-M. FerRé, «De la théâtralité des images: l'exemple du retable du «Portement de croix » de Francesco Laurana pour le roi René (1478) », dans C. Connochie-Bourgne dir., Les Arts et les Lettres en Provence au temps de René d'Anjou: analyses, rayonnement, mémoire (sous presse). Sur le retable, on peut également se référer à: Le Roi René en son temps: 1382-1481, Musée Granet - Aix-en-Provence, 11 avril - 30 septembre 1981, Aix-en-Provence, 1981, p. 163-167; F. RoBin, La Cour d'Anjou-Provence. La vie artistique sous le règne de René, Paris, 1985, p. 247-252, et H.W. KRUFT, Francesco Laurana. Ein Bildhauer der Frührenaissance, Munich, 1995, p. 176-186, 222-223 et documents XIV, XXXI, XL. 


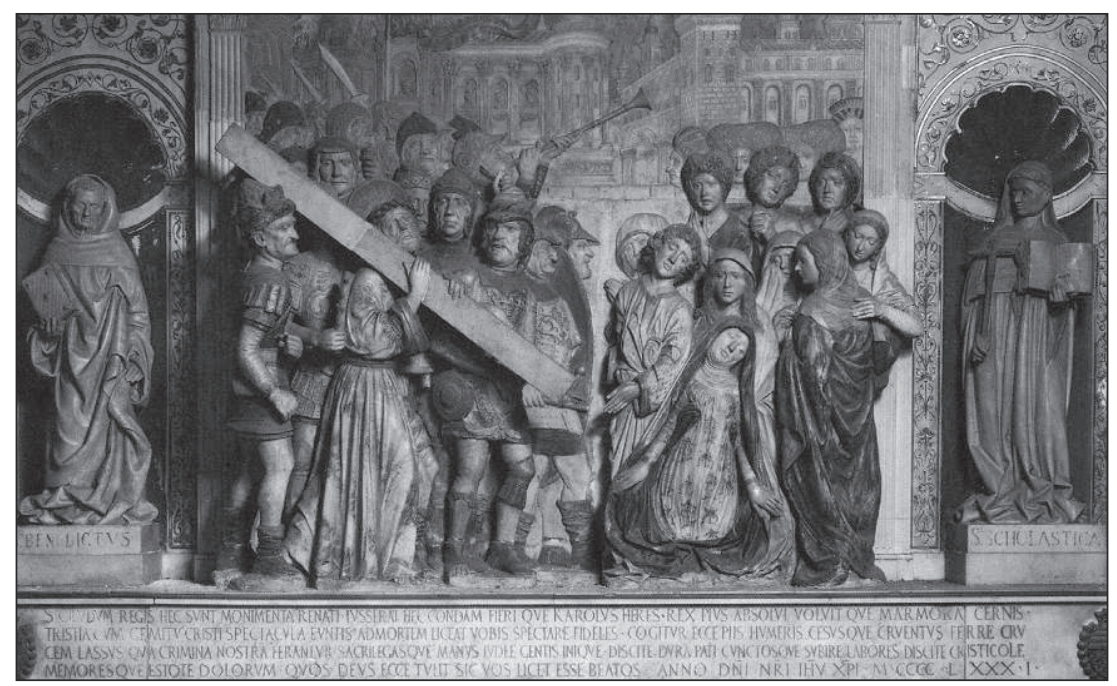

Fig. 1: Francesco Laurana, retable du Portement de Croix, 1478-1481, marbre, polychromie, 248 x $289 \mathrm{~cm}$ (pour la partie centrale), actuellement dans l'église Saint-Didier à Avignon.

s'ils s'accordent à reconnaître la scène du Portement de Croix, n'ont pas élucidé la présence de la Vierge évanouie devant le funeste cortège - l'assimilant à l'épisode de la Pâmoison lors de la Crucifixion. De même, l'aspect formel, très expressionniste, des sculptures a toujours posé problème eu égard au style habituel, délicat et serein, de l'artiste de formation italienne.

L'analyse des sources d'inspiration de Francesco Laurana ainsi que la mise en perspective des intentions du commanditaire, de l'environnement culturel et spirituel de l'œuvre ont permis néanmoins d'en révéler son sens profond. Si le retable évoque un seul et même moment du calvaire, situé avant la Crucifixion, il est une interprétation fidèle d'un extrait du Mystère de la Passion d'Arnoul Gréban - écrivain et dramaturge ayant travaillé au service de René et de son frère Charles du Maine. Cette référence au monde dramatique engage alors à s'interroger sur la réciprocité, voire la fusion, des arts au Moyen Âge.

Dans un autre domaine, l'illustration de l'exemplaire parisien du Livre du Cœur d'Amour épris écrit par René d'Anjou en 1457 pose également la question du délicat rapport entre des sources littéraires ou dramatiques et les images ${ }^{36}$. Si ce manuscrit n'est pas à proprement parler un «manuscrit dit de théâtre ${ }^{37}$, son texte

36. Ms Paris, BnF fr. 24399. La décoration de ce manuscrit aurait été exécutée dans les années 1480.

37. Voir à ce propos la typologie proposée par D. SмIтH, «Les manuscrits “de théâtre”. Introduction 


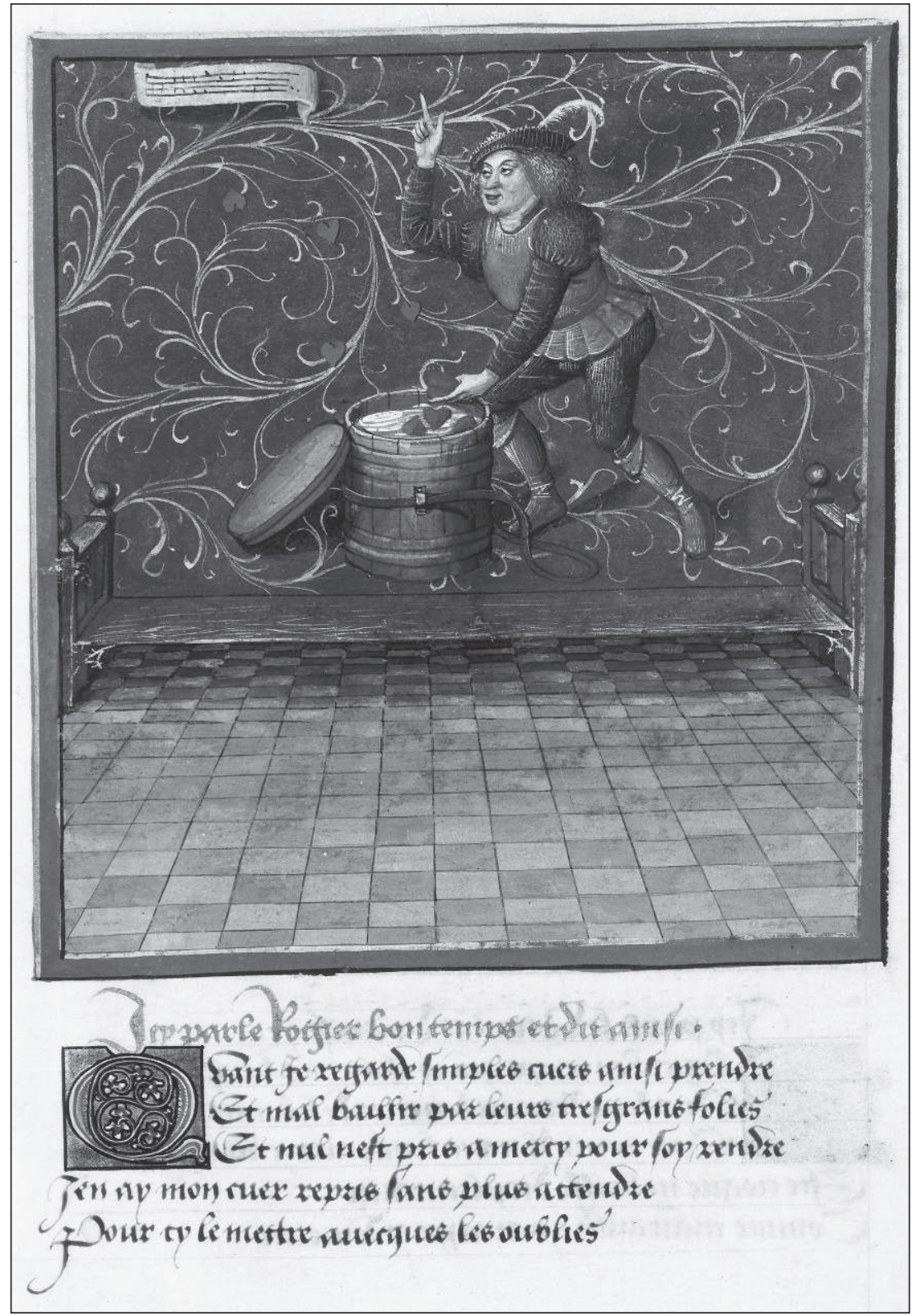

Fig. 2: René d'Anjou, Livre du Cour d'Amour épris, Paris, BnF, ms. fr. 24399, «Roger Bon Temps », fo 124. 
et ses peintures renvoient indéniablement à une théâtralité qui permet une réelle efficacité démonstrative. Les peintures de la séquence descriptive des tapisseries de Vénus (fol. 121-124 $\mathrm{v}^{\circ}$ ) présentent alors au lecteur/spectateur un vrai théâtre pour le motif du cœur, protagoniste de cette épopée courtoise. Les images se nourrissent ici du monde dramatique en faisant clairement allusion à la Farce de la Pipée et à un personnage du théâtre allégorique, Roger Bon Temps ${ }^{38}$ (Fig. 2).

D'un point de vue méthodologique, ces cas permettent en outre de prolonger les réflexions engagées par É. Mâle et de fournir, une centaine d'années plus tard, la réponse à ses questionnements sur le dialogue entre les arts. Toutefois, convient-il encore, tout en poursuivant les intentions de ce savant, de nuancer quelque peu les choses.

En effet, alors que l'iconographie traditionnelle estime par exemple que la signification est fixée dans l'objet lui-même, il faudrait peut-être aujourd'hui considérer certes plus précisément les paramètres de la création de l'œuvre (attentes et culture du commanditaire, intentions et expériences de l'auteur et de l'artiste), mais également prendre en considération l'interaction entre l'œuvre d'art et le lecteur et/ou le spectateur qui finalement construit du sens.

Un artiste qui fait référence à l'art dramatique dans son œuvre ou qui mobilise tout autre langage que le sien exclut donc une vision univoque et frontale. Il joue sur la compétence du futur observateur pour repérer des citations et des modes d'expression qui appartiennent à un autre médium et qui ouvrent des possibilités d'interprétation et de signification. Tous les créateurs de la fin du Moyen Âge semblent bien en effet réfléchir sur des nouvelles façons de dire, de voir et de penser le monde. Les écrivains conçoivent ainsi souvent leur écriture en relation avec des images - réelles ou figurées (que l'on songe par exemple à Christine de Pizan ou Guillaume de Digulleville et au développement de l'allégorie que le roi René exploite savamment) -, tandis que les peintres créent des œuvres qui forment de véritables discours.

Enfin, ces interférences entre plusieurs moyens d'expression artistique interrogent sur les démarches à mettre en place pour envisager la création médiévale. Récemment, P. Sheingorn a appelé à un effacement des frontières entre les disciplines de l'histoire de l'art, de la littérature et de l'histoire du théâtre, et a engagé à réfléchir sur ce qui constituerait une histoire de la culture visuelle d'une époque ou d'un milieu, perceptible précisément au point de convergence des études sur les images et le langage ${ }^{39}$.

codicologique à des manuscrits qui n'existent pas», Gazette du Livre Médiéval, 33, 1998, p. 1-10.

38. R.-M. Ferré, «Pour une "lecture performative" de l'œuvre de René d'Anjou? Le dialogue des arts dans le Livre du Cuer d'Amours espris de Paris : écriture, peinture, spectacle. Autour de la série des tapisseries de Vénus », dans Fl. Bouchet dir., René d'Anjou, écrivain et mécène (sous presse).

39. P. Sheingorn, «Medieval Studies and the New Art History», loc. cit., en particulier p. 143 - 
Rose-Marie FerRé - Université de Paris IV-Sorbonne, Institut National d'Histoire de l'Art, 2 rue des Petits-Champs, 75002 Paris

\section{L'art et le théâtre au Moyen Âge: jalons et perspectives}

Il revient à Émile Mâle d'avoir souligné les relations entre les arts figurés et le théâtre. Valorisant certes le document écrit et pensant la production artistique en termes d'ascendance ou de hiérarchie, ce grand penseur a cependant été attentif à l'évolution des idées et à leur transmission. Toutefois, il s'agit dorénavant de dépasser ces raisonnements traditionnels de l'influence de l'art dramatique sur les arts visuels et de favoriser une étude plus attentive des paramètres de la commande artistique. À ce titre, il paraît important de signaler les grandes étapes de la recherche sur ce sujet, longtemps resté problématique parmi les spécialistes. Mais, au-delà de ce bilan, il convient aussi de présenter les nouvelles démarches d'analyse de la création médiévale, davantage tournées vers la pluridisciplinarité et la prise en compte de la réciprocité des divers langages artistiques.

iconographie - arts - théâtre - historiographie, Émile Mâle

\section{Art and Theatre in the Middle Ages - Pointers and Perspectives}

It returns to Émile Mâle to have underlined the relations between the figurative arts and the theater. Highlighting certainly the written documents and thinking the artistic production in term of ancestry or hierarchy, this great thinker was nevertheless attentive to the evolution of the ideas and to their transmission. However, it is now advisable to exceed these traditional reasonings of the influence of the dramatic art on the visual arts and to promote a more attentive study of the parameters of the artistic command. In this way, it seems important to indicate the big stages of the research on this subject, for a long time remained problematic among the specialists. But, beyond this assessment, it is also necessary to present the new initiatives of analysis of the medieval creation, more turned to the multidisciplinarity and the consideration of the reciprocity of the diverse artistic languages.

Iconography - Arts - Drama - Historiography - Émile Mâle

146. Voir aussi les réflexions de S. BAnN, «How Revolutionary is the New Art History?», dans A.L. Rees, F. Borzello dir., The New Art History, Atlantic Highlands, 1988, p. 19-29. 
